\title{
Automatic Extraction of Anthropometric Measurements from 3D Body Scans which are Misaligned or in Natural Posture
}

\author{
Rajesh BHARTIYA*, Abhishek MISHRA, Omkar PANDE \\ 3D Measure Up, ProtoTech Solutions Pvt. Ltd., India \\ https://doi.org/10.15221/20.53
}

\begin{abstract}
Given the rise of portable scanners and the evolution of new techniques for extracting 3D body scans from photos and videos, it is becoming increasingly difficult to get body scans, which are in standard poses ('A', 'T') and aligned correctly to the standard axes. In this presentation, we discuss the challenges faced due to this and some techniques that we have applied to solve them.
\end{abstract}

Keywords: 3D body scan, measurement, alignment, apparel, anthropometry.

3D body scanning technology has enabled several use cases that were not possible a few years ago. The COVID 19 pandemic has made contactless measurements and virtual trials a necessity. A number of scanning methods are mushrooming to collect the user's body scan. This document presents the challenges that a measurement platform must address to provide reliable, consistent, and accurate measurements to produce a product that is comfortable to use.

Some of the popular use cases are

- clothes like uniforms, on-demand casuals, protective clothing or it

- fitness, medical services (posture analysis, prosthetics, wheelchairs, and mobility equipment)

- cosmetic consumables (eyeglasses, masks, gloves, helmets )

- footwear (sports like running; horse riding, fashion footwear)

The challenge of collecting reliable 3D body scans is either addressed by booth scanners, portable scanners, or mobile applications converting 2D photos to 3D models. The problem now has shifted to collecting measurements from the 3D models.

We present here the characteristics of a reliable human body measurement platform to the realization of the full potential of personalization services in the mass market.

The human body measurements platform must support variations in the file formats, scan resolutions, orientation, pose of the model, quality of the mesh, and full or partial body.

The human bodies are of many shapes and sizes. More than $2 / 3 \mathrm{rd}$ of the US population is obese or overweight.[1] Scans of these people in any pose will result in a fused mesh at points where hands or legs touch the body. The posture of the person also adds to the variation in the input. A squared shoulder or stooping posture affects where the shoulder landmark is affected. This impacts the accuracy of the shoulder width, a critical measurement for bespoke suits.

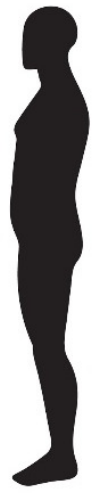

NORMAL

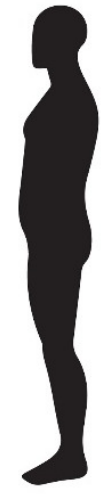

ERECT

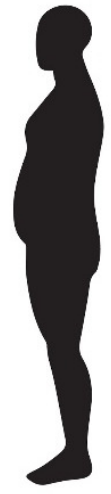

STOOPING

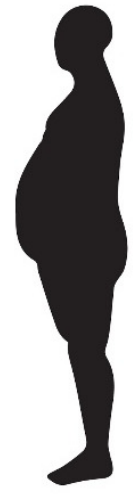

OVERWEIGHT ERECT

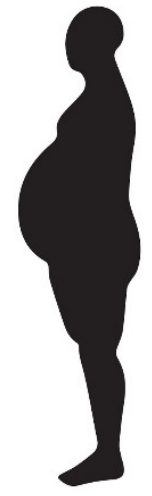

OVERWEIGHT STOPPING

* CEO, ProtoTech Solutions Pvt. Ltd., India; E-mail: 3DMeasureUp@prototechsolutions.com 
Care should be taken to train an ML model to identify the landmarks, lengths, and circumferences without bias towards the fixed poses, postures, body shapes. We present a list of poses, postures, body shapes, mesh resolutions, and defects that should be considered when training an ML model for measurements.
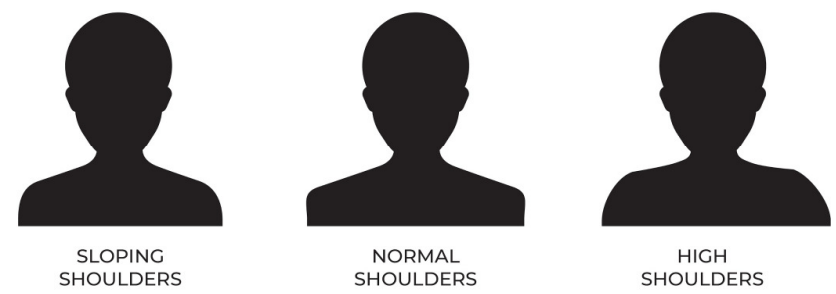

Another the $\mathrm{X}, \mathrm{Y}$, and Z-axis of each scanner may be different. The person may be facing slightly away from the camera. The scan needs to align in a way that AI or CV technology can process it. We present a simple approach to align a model in any orientation, with minimum processing time.

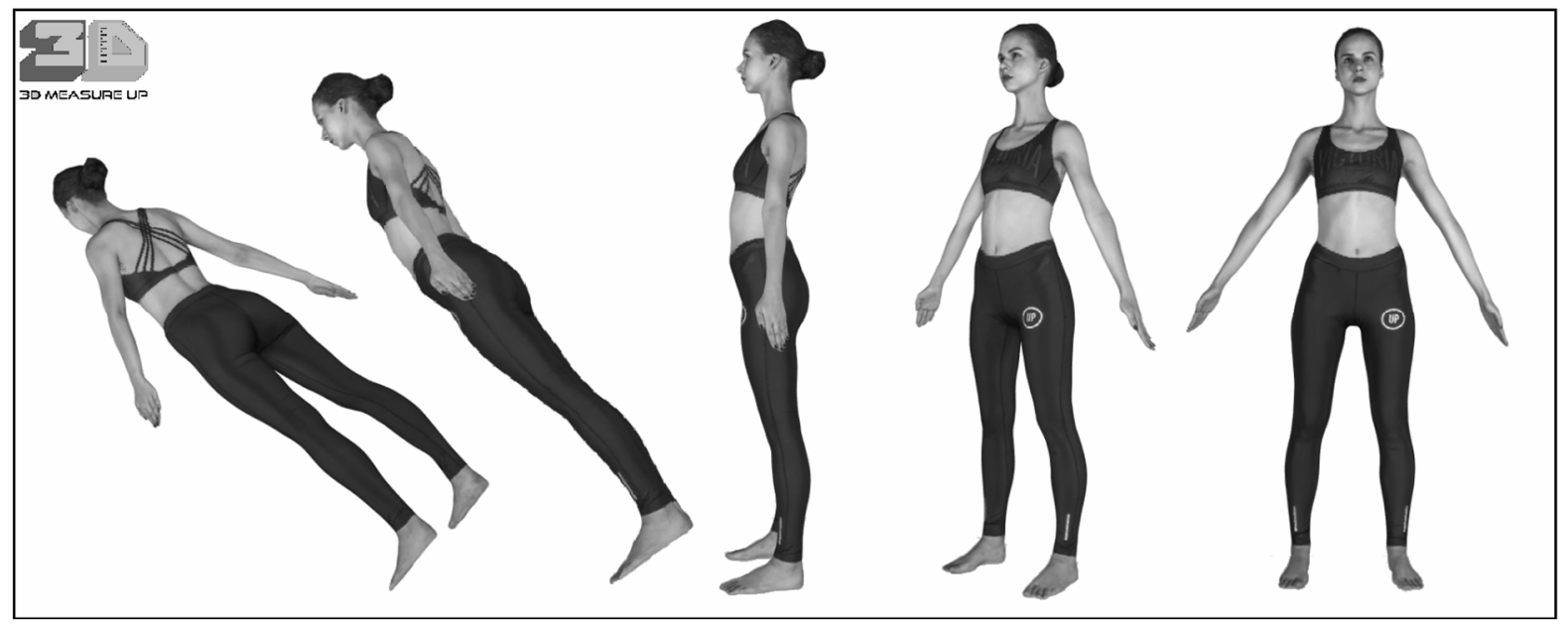

\section{Conclusion}

The measurement extraction stage of the personalization workflow ( scan - measure - design - plan produce - deliver ) is essential in determining the product experience, be it apparel, prosthetics, or posture diagnosis. A body measurement platform built for the mass market should be unbiased and accommodating.

\section{References}

[1] https://en.wikipedia.org/wiki/Obesity in the United States

[2] ISO 8559-1:2017: Size designation of clothes - Part 1: Anthropometric definitions for body measurement

[3] ISO 20685:2010: 3-D scanning methodologies for internationally compatible anthropometric databases. 